\title{
Exercising in the COVID-19 era: implications in non-alcoholic fatty liver disease (NAFLD)
}

\author{
Philip O'Gorman (D) , 1,2 Suzanne Norris ${ }^{3,4}$
}

\begin{abstract}
COVID-19 is a major public health pandemic. Risk factors for severe infection and poorer outcomes include cardiovascular disease, obesity, type 2 diabetes mellitus and nonalcoholic fatty liver disease (NAFLD). Lifestyle interventions, including diet and physical activity modifications, are the current recommended treatment for NAFLD. In this communication, the authors discuss the crossover link between NAFLD and severe COVID-19 infection and the impact of essential public health measures to suppress the spread of COVID-19 on exercise and physical activity participation in patients with NAFLD. The future of exercise prescription and the potential use of digital technology in addressing NAFLD healthcare needs in the COVID-19 era are also explored.
\end{abstract}

\section{INTRODUCTION}

COVID-19, caused by SARS-CoV-2, is a global health pandemic. ${ }^{1}$ In the absence of vaccines, public health measures such as face coverings and social distancing are the primary preventative methods of viral transmission and remain so even as vaccines roll out across the UK and Europe. While many patients with SARS-CoV-2 infection are asymptomatic or experience mild self-limiting symptoms such as fever, shortness of breath and coughing, ${ }^{2}$ severe SARS-CoV-2 infection can result in multiorgan failure and associated mortality. ${ }^{3-5}$ Risk factors associated

\footnotetext{
${ }^{1}$ School of Physiotherapy, Royal College of Surgeons in Ireland, Dublin, Ireland

${ }^{2}$ Discipline of Physiotherapy, Trinity College Dublin, Dublin, Ireland

${ }^{3}$ Department of Hepatology, St James's Hospital, Dublin, Ireland

${ }^{4}$ Department of Clinical Medicine, Trinity College Dublin, Dublin, Ireland

Correspondence to Professor Suzanne Norris, Department of Hepatology, St James's Hospital, Dublin, Ireland; SNorris@stjames.ie
}

with hospitalisation, admission to intensive treatment units and poorer outcomes include older age, male sex, non-Caucasian ethnicity ${ }^{3-5}$ and the presence of pre-existing comorbidities including cardiovascular disease (CVD), type 2 diabetes mellitus (T2DM), obesity and liver disease such as non-alcoholic fatty liver disease (NAFLD). ${ }^{5-7}$ As epidemiological data on the pandemic develops, obesity has emerged as a major risk factor, ${ }^{56}$ with patients who are obese (body mass index [BMI] $\geq 35 \mathrm{~kg} / \mathrm{m}^{2}$ ) less than 60 years old having a 2.2 fold increased likelihood of hospitalisation with SARS-CoV-2. ${ }^{8}$ This is particularly important in the context of the increasing global trends of obesity. ${ }^{9}$

\section{NAFLD AND COVID-19}

NAFLD is a global health problem and is the leading cause of chronic liver disease in North America and Europe, with one in four adults having NAFLD. ${ }^{10}$ The rising prevalence of NAFLD reflects the rising prevalence of major risks factors for NAFLD including obesity, T2DM, CVD and the metabolic syndrome. ${ }^{10}$ NAFLD is a spectrum of disease ranging from simple steatosis to nonalcoholic steatohepatitis (NASH) and cirrhosis, with approximately $25 \%$ of patients with NAFLD at risk of developing NASH. ${ }^{10}$ Given the overlap of risk factors, it is unsurprising that patients with NAFLD infected with SARS-CoV-2 have been reported to have an increased risk of severe COVID-19 outcomes. A report of 202 patients infected with COVID-19 admitted to hospital indicated that patients with NAFLD had a higher risk of COVID-19 disease progression, higher likelihood of abnormal liver function tests (LFTs) from admission to discharge and longer viral shedding time. ${ }^{11}$ Additionally, NAFLD, higher BMI, age and underlying comorbidities such as T2DM have been reported to be associated with COVID-19 progression, ${ }^{12}$ suggesting that patients with NAFLD, once infected, may have a greater risk of COVID-19 disease progression and associated complications. This increased susceptibility to severe COVID-19 infection may be linked to obesity-related impaired immune responses against the virus. ${ }^{13}$ Obesityrelated chronic inflammation, termed meta-inflammation, is characterised by pro-inflammatory responses mediated through activation of the NLR family pyrin domain-containing 3 (NLRP3) inflammasome/interleukin (IL)-1 axis. ${ }^{14}$ Additionally, adipose tissue directly secretes proinflammatory tumour necrosis factoralpha and IL-1 with subsequent downstream oxidative stress, and these pro-inflammatory pathways drive the pathogenesis in obesity-related conditions including NAFLD. ${ }^{15}$ NAFLD stage of fibrosis may also be important. In a cohort of 94 patients with NAFLD, higher fibrosis-4 and NAFLD fibrosis scores increased the likelihood of developing severe COVID-19 illness, suggesting that in the setting of NAFLD-related fibrosis, the 'cytokine storm' reported in severe COVID-19 is exacerbated. ${ }^{12}$ Furthermore, a recent paper comparing patients who are obese with NASH to patients who are obese with steatosis alone suggested that advanced stages of NAFLD may predispose patients to COVID-19 infection, indicated by markedly higher liver mRNA expression of ACE2 and transmembrane protease serine 2, genes associated with SARS-CoV-2 viral entry, in patients who are obese with NASH. ${ }^{16}$ Taken together, these data indicate that dysfunction of the innate immune response in patients who are obese may represent a key feature of the susceptibility to severe COVID-19 disease. 


\section{EXERCISE AND PHYSICAL ACTIVITY (PA) IN THE COVID-19 ERA}

Being physically active is one of the most important measures people can take to improve their health. ${ }^{17}$ The multiple benefits of exercise are well known and play an important role in CVD and cancer prevention, as well as improving traditional risk factors associated with all-cause mortality. ${ }^{18}$ Despite these proven benefits, $31 \%$ of adults worldwide are physically inactive, ${ }^{19}$ making physical inactivity (PiA) one of the leading causes of global mortality. ${ }^{17-19} \mathrm{PiA}$ is associated with many deleterious effects including loss of aerobic fitness, cognitive decline, changes in insulin signalling leading to peripheral insulin resistance, and alterations in adipose tissue lipolysis and mitochondrial pathways. Consequently, PiA has been strongly linked with many chronic diseases including CVD, obesity and NAFLD; recognised risk factors for severe COVID-19 outcomes. ${ }^{5-7} \quad$ While the benefits of exercise and PA in promoting cardiovascular health and cardiorespiratory fitness are well established, ${ }^{20}{ }^{21}$ exercise additionally alters the immune system and anti-viral responses. Animal experiments in influenza-infected mice have demonstrated that moderate exercise improves morbidity and mortality ${ }^{22}$ while conversely, intense exercise leads to poorer outcomes in response to respiratory infections. Despite these controversies and the lack of direct evidence about how $\mathrm{PA}$ and exercise might affect the immune system to alter COVID-19 susceptibility, or whether the level of physical fitness impacts the course of COVID-19 infection, these studies support the viewpoint that moderate PA maintains immunosurveillance against infectious agents.

However, public health measures to suppress COVID-19 have had significant implications for PA and exercise adherence. ${ }^{23-26}$ Localised lockdowns, social distancing and stay at home orders have forced the closure of face-to-face exercise facilities, fitness centres and public spaces, as well as suspending essential exercise trials in chronic disease populations. While acknowledging that indoor group settings for exercise may, in the absence of appropriate protection measures, confer infection risks for COVID-19 acquisition that offset the positive effects of exercise, the closure of these facilities makes being physically active more difficult. Longer term consequences of COVID-19-induced $\mathrm{PiA}$ are currently unknown but may include increased rates of sarcopenia and frailty, and increased falls risk, all of which adversely impact quality of life and contribute to disability.

Although containment measures may have introduced new barriers to being physically active, changes in work patterns and the 'work from home' advice observed in many countries may have facilitated opportunities to engage in PA. Access to online platforms delivering remote exercise classes such as yoga, pilates and high intensity interval training, with suitable exercises that could be achieved in the home environment (eg, jogging in place, burpees, rope skipping, mountain climbers, airsquats, jumping lunges, push-ups and single-step climbing) provides opportunities for maintaining or engaging in PA for some individuals. Home exercises not requiring special equipment and easily practised in the home environment include stair climbing, chair squats, sit-ups and jumping. However, in countries where physical distancing policies may feature for the short to medium term as newer COVID-19 viral strains emerge, evidence-based practical recommendations for home-based exercise to encourage and maintain PA participation will be important. ${ }^{27}$

\section{EXERCISE AND PA IN NAFLD}

Lifestyle interventions are the primary treatment for NAFLD ${ }^{28} 29$, with weight loss targets of $7 \%-10 \%$ recommended as the primary intervention endpoint. ${ }^{28}$ However, exercise-only interventions highlight the important role of exercise in patients with NAFLD, with reductions in liver fat being independent of weight loss. ${ }^{30} 31$ Reductions in intrahepatic triglyceride levels and improved insulin sensitivity have been reported with aerobic and resistance exercise, ${ }^{31} 32$ and with both moderate and high-intensity training, ${ }^{33}{ }^{34}$ suggesting that all types and intensities of exercise appear to have similar effects on liver fat and insulin sensitivity, indicating that exercise can be targeted to individual needs. Exercise-related reductions in hepatocyte lipids and insulin resistance may therefore act as a non-pharmacologic tool to address the pathophysiological mechanisms observed in NAFLD. The 'two hits' hypothesis has been the leading theory on NAFLD pathogenesis. ${ }^{35}$ The first hit is the excessive accumulation of fat in hepatocytes leading to insulin resistance. The second hit is the inflammatory response in the liver induced by reactive oxygen species and subsequent mitochondrial dysfunction and endoplasmic reticulum stress characteristic of NASH. Recent studies have challenged this hypothesis and suggest 'multiple hits' are involved in the pathogenesis of NAFLD including adipokine release, and alteration in intestinal microbiota composition and activity. ${ }^{36}$ A better understanding of how physical exercise affects liver mitochondrial function will be a key factor in the design of beneficial exercise programmes in the future. A recent paired-biopsy study reported a significant regression in histological endpoints following a 12-week aerobic exercise intervention in patients with NAFLD, ${ }^{37}$ and suggested that exercise-induced immune responses may be responsible for the observed histological benefits. This study additionally reported a potential link between histological benefit and cardiorespiratory fitness improvements. ${ }^{37} \mathrm{~A}$ recent report has suggested that higher cardiorespiratory fitness may protect against the proinflammatory response observed in severe COVID-19 and reduce the 


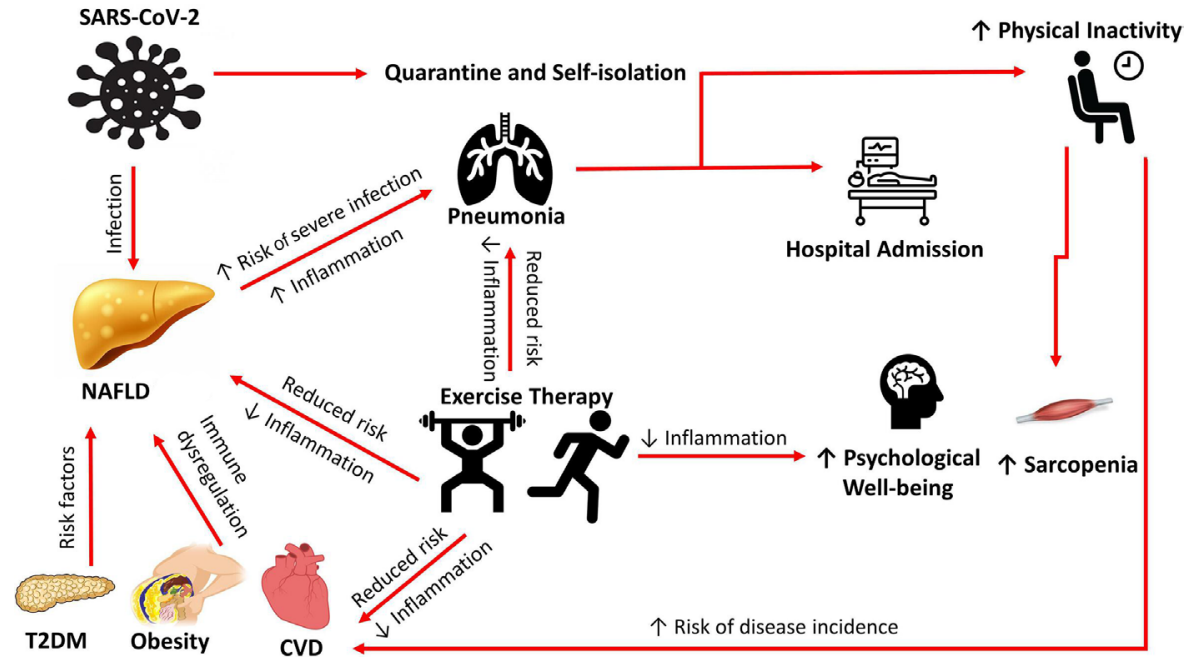

Figure 1 A summary of the benefits of exercise in NAFLD in the COVID-19 era. CVD, cardiovascular disease; NAFLD, non-alcoholic fatty liver disease; T2DM, type 2 diabetes mellitus.

risk of hospitalisation, ${ }^{38}$ suggesting that exercise-induced improvements in cardiorespiratory fitness may be protective for patients with NAFLD in the COVID-19 era. However, in the absence of primary data on the beneficial effects of exercise training programmes demonstrating a definitive protective effect against COVID-19, this remains speculative. Additional to the hepatic benefits of exercise and PA, being physically active improves extrahepatic manifestations of NAFLD, particularly the cardiovascular sequelae, ${ }^{39}$ the leading cause of mortality in this population. Consequently, PA and exercise should be promoted in order to address both the hepatic and extrahepatic manifestations of NAFLD. A summary of the benefits of exercise in patients with risk factors for the metabolic syndrome resulting in NAFLD is detailed in figure 1 .

Despite the beneficial effects of PA, patients with NAFLD engage in less PA compared with controls. Patients with NAFLD report multiple existing barriers to engaging in PA including lack of willpower, time and energy, ${ }^{40}$ and longitudinal adherence to $\mathrm{PA}$ is frequently not achieved in the community setting. COVID-19 brings additional barriers ${ }^{23}$ that make it increasingly difficult for patients with NAFLD to become and remain physically active. Furthermore, it has been argued that exercise guideline recommendations lack the high-quality evidence provided by randomised controlled trials (RCTs). This is particularly relevant in NAFLD populations where there are currently no specific detailed recommendations for exercise prescription.

\section{THE FUTURE OF EXERCISE AND PA IN NAFLD}

Exercise represents an inexpensive, democratic and effective management strategy for NAFLD in the COVID-19 era. Systems-based interventions at a population level are needed to ensure effective strategies are developed to promote exercise engagement during and beyond the COVID-19 pandemic. With the uncertainty about the resumption of traditional exercise programmes, the importance of digital health for chronic disease management is becoming more evident. ${ }^{41}$ Digital solutions such as home-based exercise training have been proven to be an effective tool in cardiac rehabilitation, improving both clinical outcomes and adherence rates. ${ }^{42}$ Similarly, studies investigating the use of digital tools in NAFLD management have reported positive and sustainable benefits. ${ }^{43-46}$
Huber et al reported improvements in non-invasive markers of hepatic fibrosis and cardiorespiratory fitness following an 8-week, web-based exercise intervention in biopsy-proven patients with NAFLD, with the benefits sustained longitudinally. ${ }^{43}$ Similarly, a 2-year, web-based lifestyle intervention was as effective as a group-based lifestyle intervention in reducing BMI and improving LFTs and surrogate markers of liver fat in patients with NAFLD. ${ }^{45}$ More recently, outcomes from a 6-month RCT using a mobile application to achieve weight loss reported that patients with NAFLD who used the mobile application had a fivefold higher likelihood of achieving $\geq 5 \%$ weight loss compared with the control group. ${ }^{46}$ These positive results support a call for large-scale studies of digital health and exercise prescription in patients with risk factors for cardiometabolic disease such as NAFLD.

At a wider societal level, the rapid development of digital health technology, the increasing trend for wearable fitness devices and the use of smartphone applications (apps) which promote PA have the potential to be a long-term enabler of PA in the management of cardiometabolic disease. Digital health technology eliminates many barriers frequently associated with traditional exercise programmes, thereby potentially reaching those in greatest need. PA apps can be tailored to individual use or group use, and can be used in small spaces during lockdown. To understand which app features help people remain physically active, an analysis of PA apps reported two broad features: motivational features which emphasise social and self-regulation of PA (eg, feedback, social support and goal setting); and educational features which focus on PA tutoring (eg, instructions, coaching and learning). ${ }^{47}$ Furthermore, gamification-related features-the use of game design elements such as points, levels and badges to make the experience more enjoyable-are increasingly 
being used in PA apps to help individuals improve health and fitness. ${ }^{48}$ A recent US study on PA app usage in 431 adults (47\% overweight or obese) who self-reported PA levels before and during lockdown identified an $18.2 \%$ reduction in $\mathrm{PA}$ levels during COVID-19 lockdown, but noted that gamification-related app features helped to buffer the decline in $\mathrm{PA},{ }^{49}$ suggesting specific app features may be more helpful than others in maintaining PA during the COVID-19 pandemic. These emerging data may help to focus strategies to address the exercise needs of specific groups of adults who have reported a negative change in exercise behaviour as a consequence of COVID-19 containment strategies. ${ }^{26}$

Exercise prescription using digital health tools for patients with NAFLD will require a systems-based approach to be implemented into practice and health policy. Exercise prescription should be designed at the individual level, incorporate behavioural change techniques, be self-monitored and eliminate barriers associated with exercise to promote long-term sustainability and engagement. Before commencement of a training regime using digital tools, exercise-naïve individuals and people with pre-existing health problems will require assessment with their primary care provider or an exercise professional, and online supervision may be recommended for individuals with little experience in exercise training, facilitated by remote monitoring of data from wearable devices and smartphone apps. Such an approach will require collaboration between policymakers, healthcare providers and stakeholders from the digital world. The COVID-19 era represents a unique opportunity to develop a systems-based approach to optimise exercise and PA engagement in patients with risk factors for cardiometabolic diseases, not just to help patients through the pandemic, but also to reduce the morbidity and mortality of cardiometabolic diseases once the pandemic has passed.

\section{RECOMMENDATIONS AND CONCLUSION}

Exercise and PA in NAFLD are increasingly more important in a world where essential public health measures to reduce the transmission of COVID-19 have exacerbated poor lifestyle behaviours which already contribute to the PiA pandemic. Exercise and PA are effective strategies for improving hepatic and extrahepatic features of NAFLD, and may provide protection against developing severe COVID-19 outcomes if infected. However, due to the lack of primary data on the beneficial effects of exercise training programmes against COVID-19, these data should be interpreted with caution. A prescription of at least 150 minutes of moderate-to-vigorous intensity aerobic exercise per week, accumulated over at least three days, is recommended in NAFLD. Resistance exercise involving all major muscle groups is recommended on two or more days per week. Patients should start exercising at low intensities and gradually build up the exercising load to reduce the likelihood of musculoskeletal injuries. Exercise prescription should be provided at the patient level by trained exercise specialists to address individualised needs and maximise adherence. This should be supported by a digital health framework to provide remote supervision and guidance to encourage long-term exercise habits during and after COVID-19.

Contributors PO'G and SN planned the paper. PO'G and SN drafted the original paper. $\mathrm{PO}$ 'G and SN revised the paper. All authors agreed with the final version of the paper.

Funding The authors have not declared a specific grant for this research from any funding agency in the public, commercial or not-for-profit sectors.

Competing interests None declared.

Patient consent for publication Not required.

Provenance and peer review Not commissioned; externally peer reviewed.

Open access This is an open access article distributed in accordance with the Creative Commons Attribution Non Commercial (CC BY-NC 4.0) license, which permits others to distribute, remix, adapt, build upon this work non-commercially, and license their derivative works on different terms, provided the original work is properly cited, appropriate credit is given, any changes made indicated, and the use is non-commercial. See: http://creativecommons.org/ licenses/by-nc/4.0/

C Author(s) (or their employer(s)) 2021. Re-use permitted under CC BY-NC. No commercial re-use. See rights and permissions. Published by BMJ.

\section{Check for updates}

To cite 0'Gorman P, Norris S. BMJ Open Gastro 2021;0:e000568. doi:10.1136/ bmjgast-2020-000568

BMJ Open Gastro 2021;0:e000568. doi:10.1136/bmjgast-2020-000568

ORCID iD

Philip 0'Gorman http://orcid.org/0000-0002-89481829

\section{REFERENCES}

1 Coronaviridae Study Group of the International Committee on Taxonomy of Viruses. The species severe acute respiratory syndrome-related coronavirus: classifying 2019-nCoV and naming it SARS-CoV-2. Nat Microbiol 2020;5:536-44.

2 Gandhi M, Yokoe DS, Havlir DV. Asymptomatic transmission, the Achilles' heel of current strategies to control Covid-19. N Engl J Med 2020;382:2158-60.

3 Guan W-J, Ni Z-Y, Hu Y, et al. Clinical characteristics of coronavirus disease 2019 in China. N Engl J Med 2020;382:1708-20.

4 Richardson S, Hirsch JS, Narasimhan $\mathrm{M}$, et al. Presenting characteristics, comorbidities, and outcomes among 5700 patients hospitalized with COVID-19 in the new York City area. JAMA 2020;323:2052-9.

5 Williamson EJ, Walker AJ, Bhaskaran K, et al. Factors associated with COVID-19related death using OpenSAFELY. Nature 2020;584:430-6 https://www.nature.com/ articles/s41586-020-2521-4

6 Ho FK, Celis-Morales CA, Gray SR, et al. Modifiable and non-modifiable risk factors for COVID-19, and comparison to risk factors for influenza and pneumonia: results from a UK Biobank prospective cohort study. BMJ Open 2020;10:e040402.

7 Zhu L, She Z-G, Cheng X, et al. Association of blood glucose control and outcomes in patients with covid-19 and pre-existing type 2 diabetes. Cell Metab 2020;31:1068-77.

8 Lighter J, Phillips M, Hochman S, et al. Obesity in patients younger than 60 years is a risk factor for COVID-19 hospital admission. Clin Infect Dis 2020;71:896-7.

9 NCD Risk Factor Collaboration (NCDRisC). Trends in adult body-mass index in 200 countries from 1975 to 2014: a pooled analysis of 1698 population-based measurement studies with $19 \cdot 2$ million participants. Lancet 2016;387:1377-96.

10 Younossi ZM, Koenig AB, Abdelatif D, et al. Global epidemiology of nonalcoholic fatty liver disease-Meta-analytic assessment of prevalence, incidence, and outcomes. Hepatology 2016;64:73-84 
11 Qin E, Qin E, Xu J, et al. Non-alcoholic fatty liver diseases in patients with COVID-19: A retrospective study. $J$ Hepatol 2020;73:451-3.

12 Targher G, Mantovani A, Byrne CD, et al. Risk of severe illness from COVID-19 in patients with metabolic dysfunction-associated fatty liver disease and increased fibrosis scores. Gut 2020;69:1545-7.

$13 \mathrm{Kim}$ J, Nam J-H. Insight into the relationship between obesity-induced low-level chronic inflammation and COVID-19 infection. Int $J$ Obes 2020;44:1541-2.

14 Vandanmagsar B, Youm Y-H, Ravussin A, et al. The NLRP3 inflammasome instigates obesity-induced inflammation and insulin resistance. Nat Med 2011;17:179-88.

15 Wree A, McGeough MD, Peña CA, et al. NLRP3 inflammasome activation is required for fibrosis development in NAFLD. J Mol Med 2014;92:1069-82.

16 Fondevila MF, Mercado-Gómez M Rodríguez $A$, et al. Obese patients with NASH have increased hepatic expression of SARS-CoV-2 critical entry points. J Hepatol 2021;74:469-71.

17 Bull FC, Al-Ansari SS, Biddle S, et al. World Health organization 2020 guidelines on physical activity and sedentary behaviour. Br J Sports Med 2020;54:1451-62.

18 Pedersen BK, Saltin B. Exercise as medicine - evidence for prescribing exercise as therapy in 26 different chronic diseases. Scand J Med Sci Sports 2015;25:1-72.

19 Kohl HW, Craig CL, Lambert EV, et al. The pandemic of physical inactivity: global action for public health. Lancet 2012;380:294-305.

20 Saint-Maurice PF, Troiano RP, Bassett DR, et al. Association of daily step count and step intensity with mortality among US adults. JAMA 2020;323:1151-60.

21 Mandsager K, Harb S, Cremer P, et al. Association of cardiorespiratory fitness with long-term mortality among adults undergoing exercise treadmill testing. JAMA Netw Open 2018;1:e183605.

22 Sim Y-J, Yu S, Yoon K-J, et al. Chronic exercise reduces illness severity, decreases viral load, and results in greater anti-inflammatory effects than acute exercise during influenza infection. $J$ Infect Dis 2009;200:1434-42.

23 Hall G, Laddu DR, Phillips SA, et al. A tale of two pandemics: how will COVID-19 and global trends in physical inactivity and sedentary behavior affect one another? Prog Cardiovasc Dis 2021;64:108-110.

24 Ammar A, Brach M, Trabelsi K, et al. Effects of COVID-19 home confinement on eating behaviour and physical activity: results of the ECLB-COVID19 international online survey. Nutrients 2020;12:1583.

25 Meyer J, McDowell C, Lansing J, et al. Changes in physical activity and sedentary behavior in response to COVID-19 and their associations with mental health in 3052 us adults. Int J Environ Res Public Health 2020;17:6469-82.

26 Faulkner J, O'Brien WJ, McGrane B, et al. Physical activity, mental health and wellbeing of adults during initial COVID-19 containment strategies: a multi-country cross-sectional analysis. J Sci Med Sport 2021:24:320-326.

27 Schwendinger F, Pocecco E. Counteracting physical inactivity during the COVID-19 pandemic: evidencebased recommendations for home-based exercise. Int J Environ Res Public Health 2020;17:3909.

28 Vilar-Gomez E, Martinez-Perez Y, Calzadilla-Bertot L, et al. Weight loss through lifestyle modification significantly reduces features of nonalcoholic steatohepatitis. Gastroenterology 2015;149:367-78. e365.

29 Gepner Y, Shelef I, Komy O, et al. The beneficial effects of Mediterranean diet over low-fat diet may be mediated by decreasing hepatic fat content. J Hepatol 2019;71:379-88

30 Keating SE, Hackett DA, Parker HM, et al. Effect of aerobic exercise training dose on liver fat and visceral adiposity. $J$ Hepatol 2015;63:174-82.

31 Hallsworth K, Fattakhova G, Hollingsworth $\mathrm{KG}$, et al. Resistance exercise reduces liver fat and its mediators in non-alcoholic fatty liver disease independent of weight loss. Gut 2011;60:1278-83.

32 Houghton D, Thoma C, Hallsworth K, et al. Exercise Reduces Liver Lipids and Visceral Adiposity in Patients With Nonalcoholic Steatohepatitis in a Randomized Controlled Trial. Clin Gastroenterol Hepatol 2017;15:96-102.

33 Zhang $\mathrm{HJ}, \mathrm{He}$ J, Pan LL. Effects of moderate and vigorous exercise on nonalcoholic fatty liver disease, a randomised clinical trial. JAMA Int Med 2016;176:1074-82.

34 Sargeant JA, Bawden S, Aithal GP, et al. Effects of sprint interval training on ectopic lipids and tissue-specific insulin sensitivity in men with non-alcoholic fatty liver disease. Eur J Appl Physiol 2018;118:817-28.

35 Csak T, Ganz M, Pespisa J. Fatty acids and endotoxin activate inflammasome in hepatocytes which release danger signals to activate immune cells in steatohepatitis. Hepatol 2011;54:133-44.

36 Buzzetti E, Pinzani M, Tsochatzis EA. The multiple-hit pathogenesis of non-alcoholic fatty liver disease (NAFLD). Metabolism 2016;65:1038-48.
37 O'Gorman P, Naimimohasses S, Monaghan A, et al. Improvement in histological endpoints of MAFLD following a 12-week aerobic exercise intervention. Aliment Pharmacol Ther 2020;52:1387-98.

38 Brawner CA, Ehrman JK, Bole S. Maximal exercise capacity is inversely related to hospitalization secondary to coronavirus disease 2019. Mayo Clinic Proc 2021;96:32-9.

39 Hallsworth $\mathrm{K}$, Thoma $\mathrm{C}$, Hollingsworth $\mathrm{KG}$, et al. Modified high-intensity interva training reduces liver fat and improves cardiac function in non-alcoholic fatty liver disease: a randomized controlled trial. Clin Sci 2015;129:1097-105.

40 O'Gorman P, Monaghan A, McGrath $M$, et al. Determinants of physical activity engagement in patients with nonalcoholic fatty liver disease: the need for an individualized approach to lifestyle interventions. Phys Ther 2021;101:1-11.

41 Ting DSW, Carin L, Dzau V, et al. Digital technology and COVID-19. Nat Med 2020;26:459-61.

42 Rawstorn JC, Gant N, Direito A, et al. Telehealth exercise-based cardiac rehabilitation: a systematic review and meta-analysis. Heart 2016;102:1183-92.

43 Huber Y, Pfirrmann D, Gebhardt I, et al. Improvement of non-invasive markers of NAFLD from an individualised, web-based exercise program. Aliment Pharmacol Ther 2019;50:930-9. 2019.

44 Pfirrmann D, Huber Y, Schattenberg JM, et al. Web-based exercise as an effective complementary treatment for patients with nonalcoholic fatty liver disease: intervention study. J Med Internet Res 2019;21:e11250.

45 Mazzotti A, Caletti MT, Brodosi L, et al. An Internet-based approach for lifestyle changes in patients with NAFLD: two-year effects on weight loss and surrogate markers. J Hepatol 2018;69:1155-63.

46 Lim SL, Johal J, Ong KW, et al. Lifestyle intervention enabled by mobile technology on weight loss in patients with nonalcoholic fatty liver disease: randomized controlled trial. JMIR Mhealth Uhealth 2020;8:e14802.

47 Conroy DE, Yang C-H, Maher JP. Behavior change techniques in top-ranked mobile apps for physical activity. Am J Prev Med 2014;46:649-52.

48 Edwards EA, Lumsden J, Rivas C, et al. Gamification for health promotion: systematic review of behaviour change techniques in smartphone apps. BMJ Open 2016;6:e012447.

49 Yang Y, Koenigstorfer J. Determinants of physical activity maintenance during the Covid-19 pandemic: a focus on fitness apps. Trans/ Behav Med 2020;10:835-42. 